\title{
Application of the Degenerated Timoshenko Beam Element Using the Adaptively Shifted Integration Technique to Elastic-Plastic Analyses Considering Large Displacements/Rotations*
}

\author{
Tomoshi MIYAMURA** and Yutaka TOI***
}

\begin{abstract}
Appropriate positions of plastic hinges can be evaluated by using the adaptively shifted integration (ASI) technique in the elastic-plastic analyses of framed structures. In this paper, the ASI technique is applied to the degenerated Timoshenko beam element developed by Dvorkin et al., which is considering the effect of large rotations/ displacements. By slightly modifying the shape functions of the Dvorkin's element, the effect of large rotations can be precisely taken into account even when the ASI technique is used. Therefore, precise rigid body rotation about a plastic hinge can be represented by the proposed element. Stresses at the hinge are also evaluated appropriately.
\end{abstract}

Key Words: Plasticity, Plastic Hinge, Finite Element Method, Structural Analysis, Framed Structure, Finite Deformation Theory, Large Rotation, ASI Technique, Degenerated Beam Element

\section{Introduction}

The plastic hinge method is often used in the elastic-plastic analysis of framed structures; i.e., the elastic deformation is represented using the beam theory, and the plastic deformation is represented by a rigid rotation about a plastic hinge. The finite element method (FEM), however, is not suitable for analyzing such discontinuous deformation due to the forming of the plastic hinge because finely subdivided analysis model is needed to analyze the plastic hinges.

Toi and Isobe ${ }^{(2)}$ proposed the adaptively shifted integration (ASI) technique to overcome such difficulty in finite (beam) element codes. The ASI technique is based on the equivalence of some beam elements and

* Received 30th October, 1998. Japanese original: Trans. Jpn. Soc. Mech. Eng., Vol. 64, No.619, A (1998), p. 666-674 (Received 19th June, 1997)

** Faculty of Engineering (formerly Institute of Industrial Science), University of Tokyo, 7-3-1 Hongo, Bunkyo-ku, Tokyo 113-8656, Japan. E-mail: miyamura @ garlic.q.t.u-tokyo.ac.jp

*** Institute of Industrial Science, University of Tokyo, 7 -22-1 Roppongi, Minato-ku, Tokyo 106-8558, Japan the rigid bodies-spring models (RBSM) ${ }^{(1)}$. By using this technique, the plastic hinges can be adaptively located at appropriate positions in the element by simply shifting the Gauss integration station.

Toi and Yang ${ }^{(3),(4)}$ applied this technique to the crush analyses of framed structures, although positions of the integration stations were prescribed before the analyses. They used the linear Timoshenko elements formulated by the total or updated Lagrangian method. When the updated Lagrangian formulation combined with the moving coordinate system was adopted, large displacements/rotations problems could be analyzed successfully. Toi and Isobe $^{(7)}$ applied this element to the explosive demolition analysis. They also carried out the buckling analysis ${ }^{(5)}$.

In this study, the ASI technique is applied to the degenerated beam element proposed by Dvorkin et al. ${ }^{(1)}$ that is considering large displacements/rotations. This element may be a beam element widely used. Some modifications are, however, necessary to apply the ASI technique to this element, which is described in the following Section 4. 


\section{The Adaptively Shifted Integration (ASI) Technique}

\subsection{The shifted integration technique}

$\mathrm{To}^{(1)}$ found that the linear Timoshenko beam element with one point Gauss integration is equivalent to the one dimensional RBSM with rotational and shear springs. As shown in Fig. 1, when the integration station in the beam element is located at $s^{1}$, and the springs in the RBSM is located at $-s^{1}$, the beam element and the RBSM are equivalent. Therefore, the plastic hinge at arbitrary position can be modeled with the beam element by simply shifting the integration station. Note that the position of the integration station is located at the opposite side of the springs (i.e., the hinge) in the corresponding RBSM. In another words, the stresses computed at the shifted integration station are physically the stresses at the opposite side about the center of the element.

Toi also found the equivalence of the cubic Euler beam and the RBSM with two rotational springs ${ }^{(1)}$, but in this study only the linear Timoshenko beam element is considered.

\subsection{The adaptively shifted integration technique}

In the shifted integration (SI) technique ${ }^{(1)}$, the positions of the integration stations are prescribed before analyses. However, the integration station should be at the Gauss point, when a member of a framed structure is in elastic. Toi and Isobe ${ }^{(2)}$ proposed the adaptive technique, i.e., the integration stations are initially located at the Gauss point, and they are shifted to appropriate positions when the plastic hinges are formed. The physical meaning of the shifting is that the initial RBSM is replaced by the another RBSM as shown in Fig. 2.

\subsection{Application of the ASI technique to the geometrically nonlinear problem}

Toi showed the equivalence of the linear Timoshenko beam element and the RBSM under the assumption that the deformation is infinitesimal and the initial configuration of the beam is straight. It is also easy to prove that this equivalence holds when the initial axial stress resultant exists, because the axial stress resultant does not depend on the coordinate $s^{1}$ in the linear Timoshenko element. Hence, the shifted integration technique can be applied to the problems with the initial axial stresses when the deformations are small.

In large displacements/rotations problems, however, the equivalence does not hold. A technique to apply the ASI technique to such problem is described in the following Section 4.

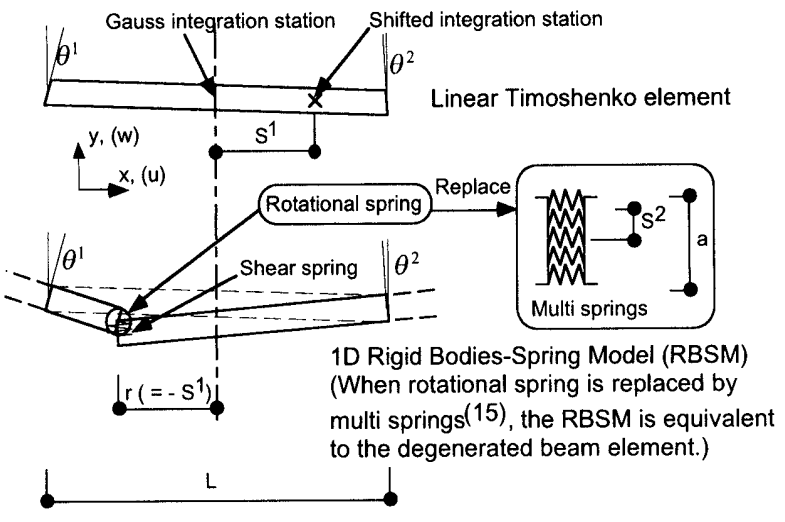

Fig. 1 Equivalence between the linear Timoshenko beam element and 1D RBSM

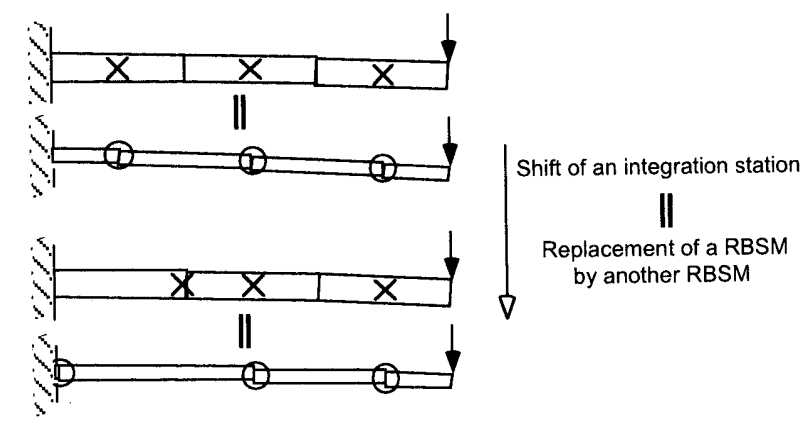

Fig. 2 Shift of an integration station and replacement of a RBSM by another RBSM

\section{Application of the ASI Technique to the Dvorkin's Degenerated Beam Element}

\section{1 Introduction}

In this section the ASI technique is applied to a degenerated Timoshenko beam element proposed by Dvorkin et al. ${ }^{(11)}$ that is considering arbitrary large displacements/rotations. As described in the previous chapter, the ASI technique is based on the infinitesimal deformation theory for straight beam. Therefore, when it is applied to the original Dvorkin's element, a kind of locking occurs as the rotation become large. A method to avoid this locking is explained in Section 4. In this section, first the formulation of the Dvorkin's element is summarized, then essential points to apply the ASI technique to degenerated beam elements are described.

\section{2 Formulations of Dvorkin's beam element}

Features of Dvorkin's element are as follows. (1) The directors are updated by the Argyris's finite rotation tensor. (2) The tangent stiffness is consistent with the finite rotation tensor. (3) The convective coordinate system is used. (4) The total Lagrangian formulation is adopted.

Figure 3 shows the geometry of the element and notations. Although arbitrary number of nodes can be 


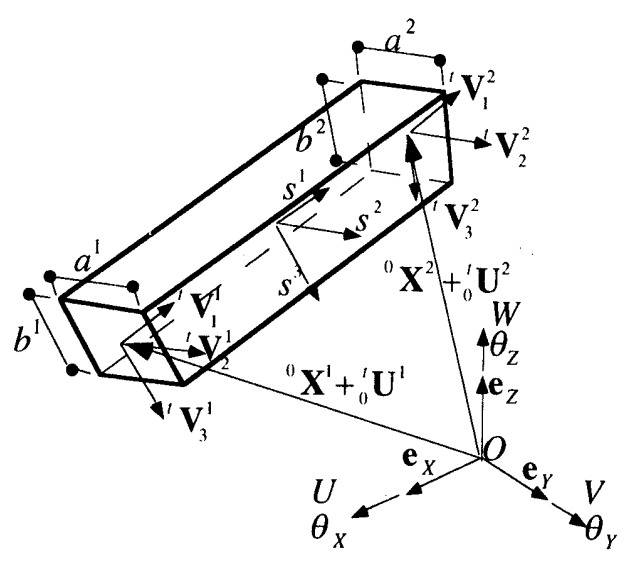

Fig. 3 Degenerated Timoshenko beam element

considered, only two-node linear element is considered since the ASI technique is employed. The initial configuration of the element is represented as,

$$
\begin{aligned}
& { }^{0} \boldsymbol{X}\left(s^{1}, s^{2}, s^{3}\right)=\sum_{n=1}^{2} N^{n}\left(s^{1}\right)\left({ }^{0} \boldsymbol{X}^{n}+\frac{a^{n}}{2} s^{20} \boldsymbol{V}_{2}^{n}\right. \\
& \left.+\frac{b^{n}}{2} s^{30} \boldsymbol{V}_{3}^{n}\right),
\end{aligned}
$$

where superscript $n$ represents a node in the element, $N^{n}\left(s^{1}\right)(n=1,2)$ are the linear shape functions, ${ }^{0} \boldsymbol{X}^{n}(n$ $=1,2)$ are the nodal position vector at the neutral axis, ${ }^{0} \boldsymbol{V}_{i}^{n}(i=2,3)$ are the two directors concerning with the cross section in each node, $a^{n}$ and $b^{n}(n=1$, 2) are widths of the cross section, and $s^{1}, s^{2}$ and $s^{3}$ are parameters of the natural coordinate system. Note that the widths of the element should be constant when the ASI technique is applied. The displacement vector from time $t$ to time $t+\Delta t$ is represented as follows :

$$
\begin{gathered}
{ }_{t}^{t+\Delta t} \boldsymbol{u}\left(s^{1}, s^{2}, s^{3}\right)=\sum_{n=1}^{2} N^{n}\left(s^{1}\right)\left\{{ }_{t}^{t+\Delta t} \boldsymbol{u}^{n}+\frac{a^{n}}{2} s^{2}{ }^{t+\Delta t} \boldsymbol{V}_{2}^{n}\right. \\
\left.\left.-{ }^{t} \boldsymbol{V}_{2}^{n}\right)+\frac{b^{n}}{2} s^{3}\left({ }^{t+\Delta t} \boldsymbol{V}_{3}^{n}-{ }^{t} \boldsymbol{V}_{3}^{n}\right)\right\},
\end{gathered}
$$

where ${ }_{t}^{\tau} \boldsymbol{u}^{n}$ is the displacement vector of neutral axis. By using the Argyris's finite rotation tensor ${ }_{t}^{\tau} \boldsymbol{R}^{n}, \mathrm{Eq}$.

(2) can be rewritten as follows:

$$
\begin{gathered}
{ }_{t}^{t+\Delta t} \boldsymbol{u}\left(s^{1}, s^{2}, s^{3}\right)=\sum_{n=1}^{2} N^{n}\left(s^{1}\right)\left\{{ }_{t}^{t+\Delta t} \boldsymbol{u}^{n}+\frac{a^{n}}{2} s^{2}{ }_{t}^{t+\Delta t} \boldsymbol{R}^{n}\right. \\
\left.\left.-\boldsymbol{I})^{t} \boldsymbol{V}_{2}^{n}+\frac{b^{n}}{2} s^{3}{ }_{t}^{t+\Delta t} \boldsymbol{R}^{n}-\boldsymbol{I}\right)^{t} \boldsymbol{V}_{3}^{n}\right\} .
\end{gathered}
$$

Details of the formulation are found in Ref.(11).

The stress and strain tensors are represented by the components of the convective coordinate system. The covariant basis vectors at time 0 and time $t+\Delta t$ are calculated as follows:

$$
\begin{aligned}
& { }^{0} \boldsymbol{G}_{i}=\frac{\partial \boldsymbol{X}}{\partial s^{i}}, \\
& { }^{t+\Delta t} \boldsymbol{g}_{i}={ }^{0} \boldsymbol{G}_{i}+\frac{\partial_{0}^{t+\Delta t} \boldsymbol{u}}{\partial s^{i}}={ }^{t} \boldsymbol{g}_{i}+\frac{\partial_{t}^{t+\Delta t} \boldsymbol{u}}{\partial s^{i}} .
\end{aligned}
$$

Increment of the Green-Lagrange strain tensor from time $t$ to time $t+\Delta t$ is calculated as follows:

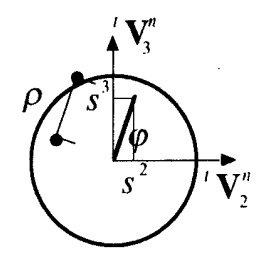

Fig. 4 Circular cross section

$$
{ }_{0} \boldsymbol{E}=\frac{1}{2}\left({ }^{t+\Delta t} \boldsymbol{g}_{i} \cdot{ }^{t+\Delta t} \boldsymbol{g}_{j}-{ }^{t} \boldsymbol{g}_{i} \cdot{ }^{t} \boldsymbol{g}_{j}\right)^{0} \boldsymbol{G}^{i} \otimes{ }^{0} \boldsymbol{G}^{j}
$$

Note that the total Lagrangian formulation is adopted in the original Dvorkin's element.

The linearized incremental equilibrium equation is calculated by substituting from Eq. ( 6 ) and increment of the stress tensor into the linearized equation of virtual work. The increment of the stress tensor is calculated by using the constitutive equations as shown in the following Section 3. 4.

\section{3 Numerical integration in the cross section}

The energy integration in the directions of $s^{2}$ and $s^{3}$ in Fig. 3 is carried out numerically. In the following illustrative examples the cross sections of beams are circular. The trapezoidal rule is used in $r$ direction and Simpson's rule in $\varphi$ direction (see Fig. 4). The number of integration points is 112 (in $r$ direction 7 , in $\varphi$ direction 16).

\section{4 Elastic-plastic constitutive equation}

Strains are assumed small although arbitrary large displacements and rotations are considered. Hence, the constitutive equations for the infinitesimal deformation can be directly employed for representing the relationship between the increment of the Green-Lagrange strain tensor and that of the second Piola-Kirchhoff stress tensor. Note that those tensors do not change when the material is subjected to a rigid body translation.

Although the elastic-plastic relation is introduced in the axial direction, the shear deformation is assumed to be elastic. The bilinear model that is considering the kinematic hardening is employed for the relationship between the Cauchy stress ${ }^{t} \sigma_{11}$ and the infinitesimal strain $\varepsilon_{11}$ as follows:

$$
{ }^{t+\Delta t} \sigma_{11}=\frac{H^{\prime}}{E+H^{\prime}}{ }^{t} \sigma_{11}+\frac{E H^{\prime}}{E+H^{\prime}} \Delta \varepsilon_{11}+\frac{E}{E+H^{\prime}}{ }^{t} \sigma_{Y 11},
$$

where subscript 1 shows the axial direction, ${ }^{t} \sigma_{11}$ is the axial stress at time $t, E$ is Young's modulus, $H^{\prime}$ is the strain hardening parameter, $\Delta \varepsilon_{11}$ is increment of the axial strain and ${ }^{t} \sigma_{Y 11}$ is the yield stress at time $t$. Equation ( 7) can be rewritten as

$$
\begin{gathered}
{ }^{t+\Delta t} \sigma_{11}=\frac{H^{\prime}}{E+H^{\prime}}\left({ }^{t} \sigma_{11}+E \Delta \varepsilon_{11}\right)+\frac{E}{E+H^{\prime}}{ }^{t} \sigma_{Y 11} \\
={\frac{H^{\prime}}{E+H^{\prime}}}^{t+\Delta t} \sigma_{11}^{T}+\frac{E}{E+H^{\prime}}{ }^{t} \sigma_{Y 11},
\end{gathered}
$$


where ${ }^{t+\Delta t} \sigma_{11}^{T}$ is the elastic predictor of stress ${ }^{t+\Delta t} \sigma_{11}$. When ${ }^{t+\Delta t} \sigma_{11}^{T}$ is smaller than ${ }^{t} \sigma_{Y 11}$, unloading occurs and ${ }^{t+\Delta t} \sigma_{11}^{T}$ is set to ${ }^{t+\Delta t} \sigma_{11}$.

\section{5 Extrapolation of stresses}

In the ASI technique, the stresses at the point other than the integration stations have to be computed to determine the appropriate positions of the plastic hinges. Such stresses, however, cannot directly be recovered in the linear beam element, since stresses are evaluated only at an integration station. Therefore, the stresses are extrapolated by using the shear stresses (refer to Ref. ( 8$)$ )). First the bending moment around the director $V_{2}^{n}$ is extrapolated by using the shear stress resultant as follows:

$$
M_{2}=M_{Q 2}+Q_{Q 13}\left(s_{\text {new }}^{1}-s^{1}\right) L,
$$

where subscript $Q$ indicates quantities in the integration station, $s^{1}$ is the position of the integration station, $s_{\text {new }}^{1}$ is the position where the stress is evaluated, $L$ is the length of the beam element, $M_{Q 2}$ and $Q_{Q 13}$ are the bending moment and the shear stress resultant at the integration station, respectively, and $M_{2}$ is the extrapolated bending moment at $s_{\text {new }}^{1}$. The bending moment around the director $V_{3}^{n}$ can be calculated similarly.

The axial stress is calculated from these bending moments and the axial stress resultant as follows:

$$
\begin{aligned}
\sigma_{11} & =\frac{N_{11}}{A}+\frac{M_{3}}{I_{3}} s^{2}+\frac{M_{2}}{I_{2}} s^{3} \\
& =\frac{N_{11}}{A}+\frac{M_{Q 3}}{I_{3}} s^{2}+\frac{M_{Q 2}}{I_{2}} s^{3}+\left(\frac{Q_{Q 12}}{I_{3}} s^{2}+\frac{Q_{Q 13}}{I_{2}} s^{3}\right) \\
& \times\left(s_{\text {new }}^{1}-s^{1}\right) L \\
& =\sigma_{Q 11}+\left(\frac{Q_{Q 12}}{I_{3}} s^{2}+\frac{Q_{Q 13}}{I_{2}} s^{3}\right)\left(s_{\text {new }}^{1}-s^{1}\right) L \\
& =\sigma_{Q 11}+\left(\frac{A \tau_{m Q 12}}{I_{3}} s^{2}+\frac{A \tau_{m Q 13}}{I_{2}} s^{3}\right)\left(s_{\text {new }}^{1}-s^{1}\right) L,
\end{aligned}
$$

where $\sigma_{11}$ is the extrapolated axial stress at $\left(s_{\text {new }}^{1}, s^{2}\right.$, $\left.s^{3}\right), \sigma_{Q 11}$ is the axial stress at the integration point $\left(s^{1}\right.$, $\left.s^{2}, s^{3}\right), N_{11}$ is the axial stress resultant, $\tau_{m Q 12}$ and $\tau_{m Q 13}$ are the shear stresses at the integration point in the neutral axis $\left(s^{1}, 0,0\right)$ and $A, I_{2}$ and $I_{3}$ are the area and the moments of inertia of the cross section, respectively. By employing the shear stresses at the neutral axis in Eq. (10), the contribution of them to the torsional stress resultant is excluded.

\section{6 Conditions for the shifting}

In degenerated beam elements, the yielding first occurs at the integration points near the surface. After that the yielding regions spread as deformation becomes large. Therefore, some criteria are necessary to judge when the plastic hinge is formed. The following conditions for the shifting of the integration station are proposed.

(1) A group of the integration points in a cross section (the integration station) is shifted simultaneously.

(2) The integration station can only be located at three positions, i.e., $s^{1}=-1,0$ or 1 .

(3) The integration station is located at a position where the number of yielded points is the largest. The points at the surface of the element are excluded in counting the number, because when the yielding occurs only at the surface, the elastic deformation dominates and the integration station should be located at the center of the element. Note that the extrapolated stress in Eq. (10) is used for the judgement of yielding at the points other than the integration points.

(4) The shifting is done only when the part of the cross section yielded, or when the whole region of the cross section becomes elastic (Eq. (10) cannot be used when yielding occurs).

(5) When the numbers of the yielding point at $s^{1}$ $=-1$ and $s^{1}=1$ is almost the same, i.e., when pure bending or axial force is applied, the integration station is placed at the center of the element.

(6) The judgement of the shifting is done at the beginning of an incremental step by monitoring the stresses calculated at the previous incremental step.

(7) When the integration station is shifted, the stiffness matrix is calculated using the extrapolated axial stresses that are calculated using Eq. (10).

(8) The shear stresses are assumed to be constant in the element.

(9) The integration stations are not shifted during the Newton-Raphson iteration.

(10) The yielding stress corresponding to a fixed set of coordinates $s^{2}$ and $s^{3}$ is assumed constant along the coordinate $s^{1}$.

\section{7 Elastic-plastic analysis of a cantilever}

The present element is evaluated by an example, which is a cantilever under a concentrated lateral load at its tip. Table 1 shows material properties and sizes. Lateral displacement is incrementally prescribed at the tip. Figure 5 illustrates the relationships between the load and the displacement. "TL-A" and "TL-C" along with the number of subdivision indicate the analysis with and without the ASI technique, respec-

Table 1 Material properties and sizes of the cantilever

\begin{tabular}{ll}
\hline $\begin{array}{l}\text { Young's modulus } E \\
\text { Strain hardening }\end{array}$ & $206 \mathrm{GPa}$ \\
parameter $H^{\prime}$ & $E / 2100$ \\
Yield stress $\sigma_{Y}$ & $294 \mathrm{MPa}$ \\
Cross section & Circular, Diameter $=70 \mathrm{~mm}$ \\
Length & $\mathrm{L}=2.0 \mathrm{~m}$ \\
\hline
\end{tabular}




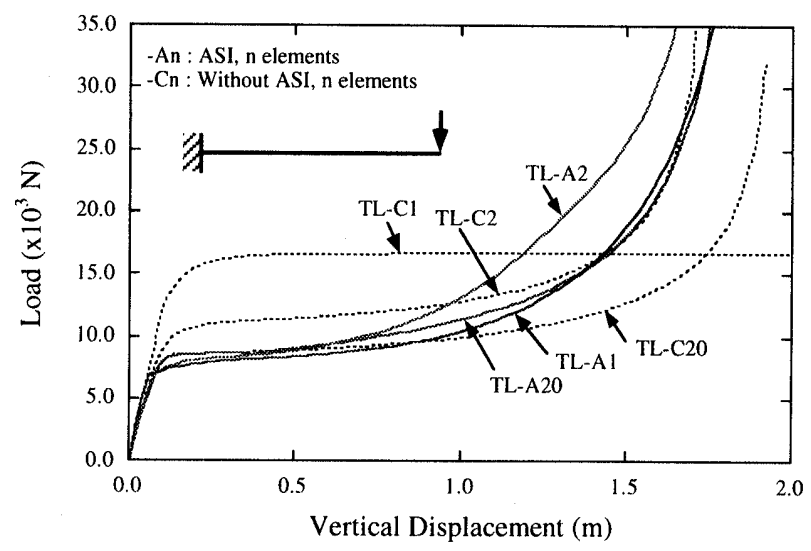

Fig. 5 The effect of the ASI technique applied to Dvorkin's element; elastic-plastic analysis of a cantilever; load vs displacement at tip

tively. When the lateral displacement is less than about $0.5 \mathrm{~m}$, the results obtained by the ASI technique are good; i.e., the load-displacement curves of TL$\mathrm{A} 1$ and $\mathrm{TL}-\mathrm{A} 2$ almost agree with the curves of $\mathrm{TL}-$ C20. When the prescribed displacement becomes larger, however, a kind of locking occurs; i.e., the results of TL-A1, TL-A2 and TL-A20 show overstiff. The result with the finely subdivided model using the ASI technique (TL-A20) is not converged to the result of TL-C20 that corresponds to the exact solution.

4. Application of the ASI Technique to the Degenerated Beam Element with Redefinition of Shape Functions

\section{1 The reason why the shape functions have to be refined}

The equivalence of the beam element and the RBSM described in Section 2 holds only when the initial configuration of the beam element is straight and the deformation is small. On the other hand, Dvorkin's beam element considers a curvature of the initial or deformed configuration. Even when the initial configuration is straight, a curvature of the deformed configuration is considered. If the linear shape function is used, the neutral axis remains straight in the deformed configuration, but the directors at nodes are not perpendicular to the neutral axis (this represent the curvature).

If the directors are redefined to be perpendicular to the neutral axis in each incremental step, the equivalence described in Section 2 holds in each incremental step. The shape functions using the directors are also redefined. This means that the beam is successively approximated by straight beam.

\section{2 The redefinition of the shape functions}

As shown in Fig. 6, at the beginning of each incremental step, the directors are redefined to be per-

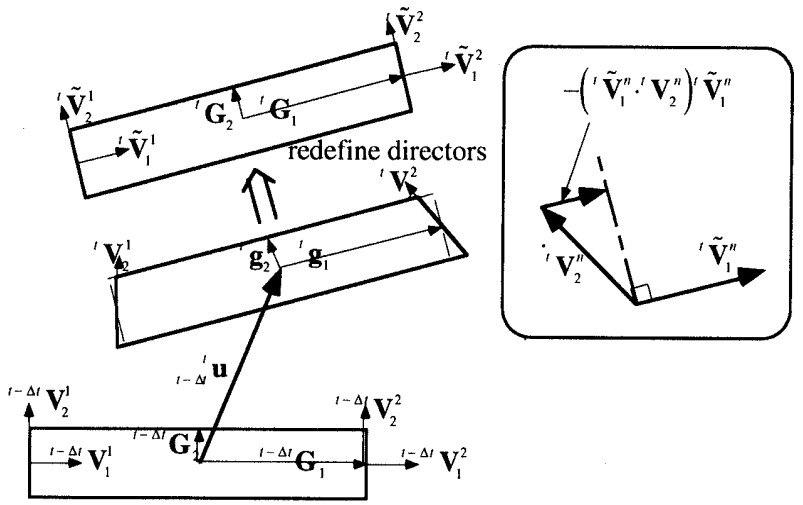

Fig. 6 Redefinition of the directors

pendicular to the current neutral axis. Figure 6 depicts two-dimensional beam element for simplicity. The redefined directors are distinguished from the old directors by " ".

The directors are redefined as follows. First, ${ }^{t} \widetilde{\boldsymbol{V}}_{1}^{n}$ is defined in the direction of the current neutral axis. Then ${ }^{t} \boldsymbol{V}_{2}^{n}$ is updated as follows:

$$
\tilde{\boldsymbol{V}}_{2}^{n}={ }^{t} \boldsymbol{V}_{2}^{n}-\left({ }^{t} \tilde{\boldsymbol{V}}_{1}^{n} \cdot{ }^{t} \boldsymbol{V}_{2}^{n}\right)^{t} \tilde{\boldsymbol{V}}_{1}^{n}
$$

If the torsional deformation occurs, the directors of two nodes, i.e., ${ }^{t} \tilde{\boldsymbol{V}}_{2}^{1}$ and ${ }^{t} \tilde{\boldsymbol{V}}_{2}^{2}$ cannot be on the same plane. Therefore, the following averaged vector is used :

$$
\left.{ }^{t} \tilde{\boldsymbol{V}}_{2}^{n}\right|_{n e w}=\frac{{ }^{t} \tilde{\boldsymbol{V}}_{2}^{1}+{ }^{t} \tilde{\boldsymbol{V}}_{2}^{2}}{\left|{ }^{t} \widetilde{\boldsymbol{V}}_{2}^{1}+{ }^{t} \tilde{\boldsymbol{V}}_{2}^{2}\right|}
$$

They are normalized to keep the width of the cross section constant. This correction does not affect the solution when the integration station is located at the center of the element. Finally, ${ }^{t} \tilde{\boldsymbol{V}}_{3}^{n}$ is calculated as follows :

$$
{ }^{t} \tilde{\boldsymbol{V}}_{3}^{n}={ }^{t} \tilde{\boldsymbol{V}}_{1}^{n} \times{ }^{t} \tilde{\boldsymbol{V}}_{2}^{n}
$$

The shape function at time $t$ is redefined by using Eqs. (11) to (13) as follows:

$$
\begin{aligned}
& { }^{t} \boldsymbol{x}\left(s^{1}, s^{2}, s^{3}\right)=\sum_{n=1}^{2} N^{n}\left(s^{1}\right)\left({ }^{0} \boldsymbol{X}^{n}+{ }_{0}^{t} \boldsymbol{U}^{n}+\frac{a^{n}}{2} s^{2 t} \tilde{\boldsymbol{V}}_{2}^{n}\right. \\
& \left.+\frac{b^{n}}{2} s^{3 t} \tilde{\boldsymbol{V}}_{3}^{n}\right) .
\end{aligned}
$$

\section{3 Redefinition of the convective coordinate system and transformation of the stress tensor}

Dvorkin's beam element is formulated by the total Lagrangian method with the use of the convective coordinate system. However, when the shape functions are redefined as described in the previous section, the convective coordinate system referring to the initial configuration is difficult to be defined. Here, the new convective coordinate system is defined by updating the basis vectors as follows :

$$
{ }_{t} \boldsymbol{G}_{i}=\frac{\partial^{t} \boldsymbol{x}}{\partial s^{i}},
$$

where the left subscript $t$ indicates that the vector is 
redefined at time $t$. Because the convective coordinate system is redefined, it is convenient to utilize the updated Lagrangian formulation. The following Green-Lagrange strain tensor referring to the configuration at time $t$ is used:

$$
{ }_{t}^{t+\Delta t} \boldsymbol{E}=\frac{1}{2}\left({ }_{t}^{t+\Delta t} \boldsymbol{g}_{i} \cdot{ }_{t}{ }^{t+\Delta t} \boldsymbol{g}_{j}-{ }_{t} \boldsymbol{G}_{i} \cdot{ }_{t} \boldsymbol{G}_{j}\right) \boldsymbol{G}_{t} \otimes_{t} \boldsymbol{G}^{j},
$$

where ${ }_{t}^{t+\Delta t} \boldsymbol{g}_{i}$ is the covariant basis vectors at time $t$ $+\Delta t$ of the convective coordinate system defined at time $t$, and ${ }_{t} \boldsymbol{G}^{i}$ is the contravariant basis vector corresponding to ${ }_{t} \boldsymbol{G}_{i}$. The increment of the above strain tensor at time $\tau$ is as follows:

$$
{ }_{i} \boldsymbol{E}=\frac{1}{2}\left({ }_{t}^{\tau+\Delta \tau} \boldsymbol{g}_{i} \cdot{ }_{t}^{\tau+\Delta \tau} \boldsymbol{g}_{j}-{ }_{t}^{\tau} \boldsymbol{g}_{i} \cdot{ }_{t}^{\tau} \boldsymbol{g}_{j}\right)_{t} \boldsymbol{G}^{i} \otimes{ }_{t} \boldsymbol{G}^{j}
$$

When large displacements/rotations are considered, but the strains are assumed small, the convective coordinate system is considered to be a co-rotational system. Because the rigid rotation dominates comparing with the other deformations in the co-rotational system, the following relation is possible (see, for example, Section 6.3.1 of Ref.(16)):

$$
{ }_{t}^{\tau} \boldsymbol{R}^{T} \cdot{ }^{\tau} \boldsymbol{T} \cdot{ }_{t}^{\tau} \boldsymbol{R} \approx{ }_{t}^{\tau} \boldsymbol{S},
$$

where ${ }_{t}^{\tau} \boldsymbol{R}$ is the rigid rotation tensor at the integration station, ${ }^{\tau} \boldsymbol{T}$ is the Cauchy stress tensor at time $\tau,{ }_{t}^{\tau} \boldsymbol{S}$ is the second Piola-Kirchhoff stress tensor at time $\tau$ referring the configuration at time $t$. If the shear deformation is small, the cross section at the integration station is almost perpendicular to the neutral axis. Therefore the basis vectors at time $\tau$ defined in Eq. (15) are approximately represented by the basis vectors at $t$ and the rotation tensor ${ }_{t}^{\tau} \boldsymbol{R}$ as follows:

$$
{ }_{\tau} \boldsymbol{G}_{i} \approx{ }_{t}^{\tau} \boldsymbol{R} \cdot{ }_{t} \boldsymbol{G}_{i} .
$$

By using Eqs. (18) and (19), and considering the reference coordinate systems of ${ }_{t}^{\tau} \boldsymbol{S}$ and ${ }^{\tau} \boldsymbol{T}$, the following relationship can be derived:

$$
{ }^{\tau} T^{i j} \approx{ }_{t}^{\tau} S^{i j}
$$

Equation (20) means that the stress in transformed by simply transferring the components of the stress tensors. Note that ${ }^{t} T^{i j}$ and ${ }_{t}^{\tau} S^{i j}$ are the components of the different coordinate systems. Because Eq. (20) is derived by using Eqs. (18) and (19) that is possible only when the lateral shear strain is small, the present element can be applied to only slender beam structures.

If the shape function is not redefined and the convective coordinate system is continuously used from time 0 , the following relationship between ${ }^{\tau} T^{i j}$ and ${ }_{t}^{\tau} S^{i j}$ can be derived (see, for example Ref.(17)) :

$$
{ }^{\tau} T^{i j}=\frac{1}{{ }_{t}^{\tau}}{ }^{\tau} S^{i j},
$$

where ${ }_{t}^{\tau} J$ is the determinant of the deformation gradient. In this case, the condition that the lateral shear strain is small is not used ; i.e., Eq. (21) can be applied to thick beams. If strains are small ${ }_{t}^{\tau} J \approx 1$ and Eq. (20) agrees with Eq. (21).

The present technique can easily be implemented by slightly modifying a code for Dvorkin's element; i.e., the subroutines that update the directors and the basis vectors using Eqs. (11) to (13) and (19) is added to the code. Other parts of the code are not changed. In the following sections, the element discussed in this section is called the "proposed element".

\section{4 Application of the ASI technique to the other elements considering large displace- ments/rotations}

Many beam elements considering large displacements and rotations were proposed. Those elements are classified as: (1) the element based on the socalled co-rotational method ${ }^{(14)}$, (2) the element using the moving coordinate combined with the updated Lagrangian formulation ${ }^{(3),(4)}$ and ( 3 ) the degenerated isoparametric element like Dvorkin's element. Since the moving coordinate system of (2) is $\mathrm{a} \mathrm{CO}^{-}$ rotational system, there exist little differences between (1) and (2). In the co-rotational method, large rotations can be considered although approximated equations are used to represent the deformations other than the rotations. Hence, this technique is often used with the stress resultant formulations.

The simplest co-rotational coordinate system for two node element is defined by a basis vector whose direction coincides with a line connecting two end nodes, and by two basis vectors whose directions are perpendicular to the line. The coordinate system is rigidly rotated with the element, and this rotation is approximately equal to the rigid rotation obtained by the polar decomposition of the deformation gradient. If the governing equation for the straight beam is used in the co-rotational coordinate system, the element is almost equivalent to the proposed degenerated element illustrated in the previous sections. Therefore, the ASI technique can be applied. Toi and Yang applied the SI or ASI technique to the linear Timoshenko element using the stress resultant formulation ${ }^{(3)}$. When the updated Lagrangian formulation is used with a moving coordinate system, good results were obtained.

On the other hand, the degenerated isotropic element like Dvorkin's element is not suitable for the ASI technique, since the curvature after deformation is strictly considered in the formulation and it is not approximated by the straight beam theory. Note that some elements based on the co-rotational method are also considering the effect of the curvature. The ASI technique is not suitable for those elements.

\section{5 Numerical tests of the proposed element}

The proposed element is tested by analyzing some examples. Figure 7 illustrates the result of "roll up" 


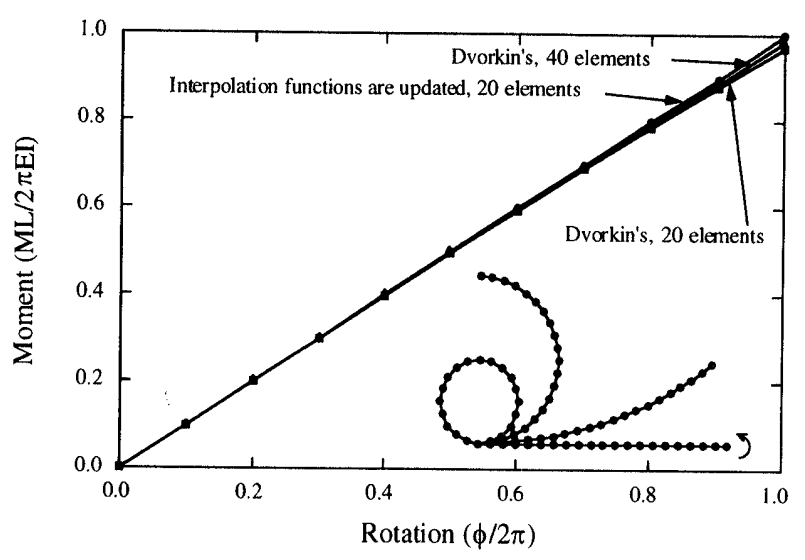

Fig. 7 Comparison between Dvorkin's element and the presented element; the roll up of an elastic cantilever (refer to Simo et al. ${ }^{(18)}$ )

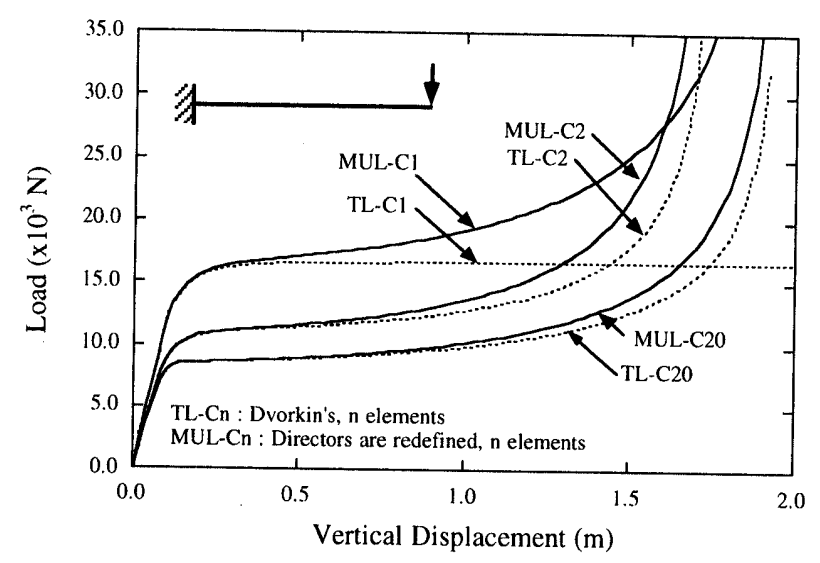

Fig. 8 Comparison between Dvorkin's element and the proposed element; elastic-palstic analysis of a cantilever

problem, which is a benchmark test of large rotation problems. The moment-rotation relationships calculated by Dvorkin's element and by the proposed element without the ASI technique, and that of the theoretical solution are shown.

The rotation moment by Dvorkin's element is a little smaller than the theoretical solution when large rotation is applied. This is because the directors at nodes are inclined to the neutral axis and the width of the cross section is reduced. This phenomenon occurs especially in lower order elements. On the other hand, the result by the proposed element agrees with the theoretical solution, because the width of the cross section is not reduced due to the updating of the directors. This characteristic is another feature of this element.

Next example is the same as in Section 3.7. Figure 8 illustrates the load and the vertical displacement relationship. 'MUL-C' indicates the proposed element without the ASI technique. The number of subdivision is attached to it. In this example, the effect of the reduction of the width is more significant,

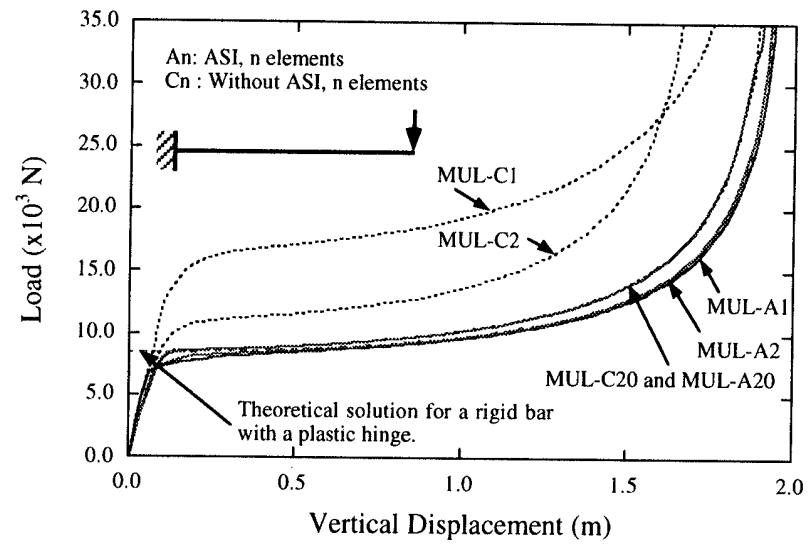

Fig. 9 The effect of the ASI technique applied to the proposed element (interpolation functions are redefined); elastic-plastic analysis of a cantilever; load vs. displacement at tip

since the deformation concentrates at the fixed boundary. The difference of the results by the Dvorkin's element and the proposed element is larger than that of the previous example.

The second example is a special case. The purpose of showing these two examples is not to point out the shortcoming of the Dvorkin's element, but to show that the proposed element can sufficiently be used as a normal beam element. Various kinds of interpolation of the director field are found in Ref.(18).

In Fig. 9, the second example is analyzed by the proposed element combined with the ASI technique that is indicated by 'MUL-A' along with the number of subdivision. In the following sections and in Table 3 , the proposed element without the ASI technique is called the 'conventional' element. The result of MULA1 almost agrees with the result of MUL-C20. The result of MUL-A1 completely agrees with the theoretical solution of a rigid bar with rotational spring. The difference of the results of MUL-A1 and MUL-C20 is due to the accuracy of elastic solution.

The result of MUL-A20 agrees with the result of MUL-C20. The solutions obtained by the conventional' element and the element with the ASI technique converge to the same values when the model is subdivided finely. Therefore, the proposed element satisfies a necessary condition for practical analyses.

A disadvantage of the proposed element with the ASI technique is that, when the strain hardening is taken into account, the yield function is not updated precisely because accuracy of the elastic solution is reduced when the integration station is shifted. For instance, in the example of a cantilever under a lateral load, the reaction force becomes smaller than exact value when the strain hardening parameter becomes larger. 


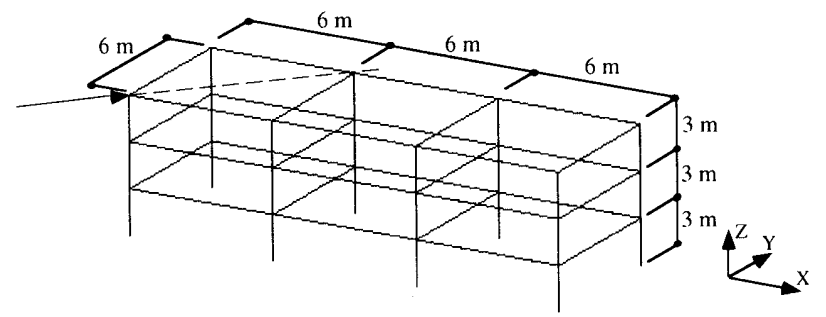

Fig. 10 Configuration of the 3D portal frame

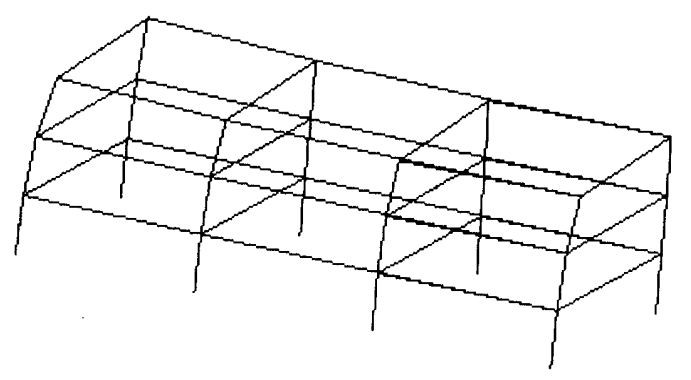

Fig. 11 Deformed configuration (model 2a, displacement $=0.8 \mathrm{~m}$ )

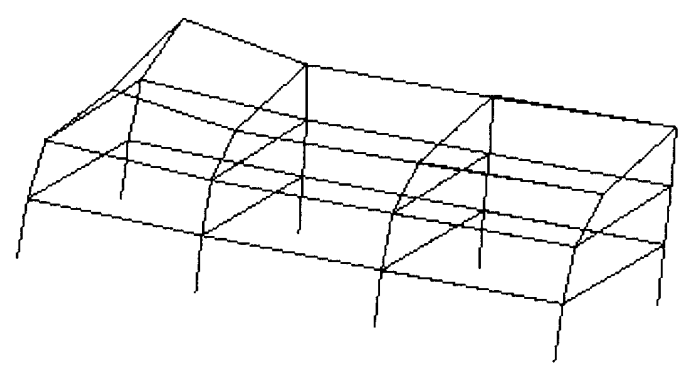

Fig. 12 Deformed configuration (model 2a, displacement $=2.0 \mathrm{~m}$ )

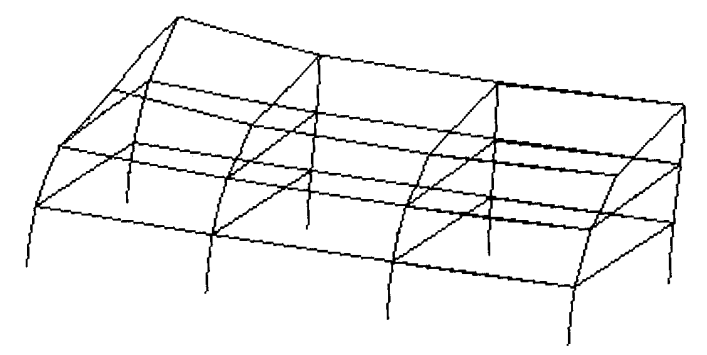

Fig. 13 Deformed configuration (model 2c, displacement $=2.0 \mathrm{~m}$ )

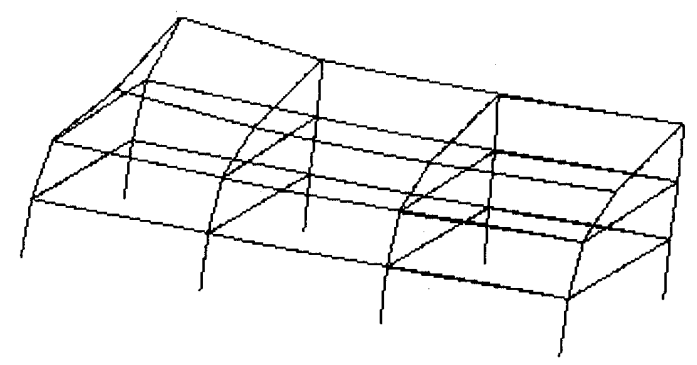

Fig. 14 Deformed configuration (model 20c, displacement $=2.0 \mathrm{~m}$ )
Table 2 Material properties and cross section's size of the $3 \mathrm{D}$ portal frame

\begin{tabular}{ll}
\hline Young's modulus & $206 \mathrm{GPa}$ \\
Strain hardening param. & $0.1 \mathrm{GPa}$ \\
Yield stress & $294 \mathrm{Mpa}$ \\
Cross section & Circular \\
& Diameter $=200 \mathrm{~mm}$ \\
\hline
\end{tabular}

Table 3 Analysis models of the 3D portal frame

\begin{tabular}{ll}
\hline model method & $\begin{array}{l}\text { number of } \\
\text { subdivisions } \\
\text { [column]-[beam] }\end{array}$ \\
\hline
\end{tabular}

\begin{tabular}{ccc}
\hline $2 \mathrm{c}$ & conventional & $2-2$ \\
$2 \mathrm{a}$ & ASI & $2-2$ \\
$20 \mathrm{c}$ conventional & $20-40$ \\
$10 \mathrm{a} \quad$ ASI & $10-10$ \\
\hline
\end{tabular}

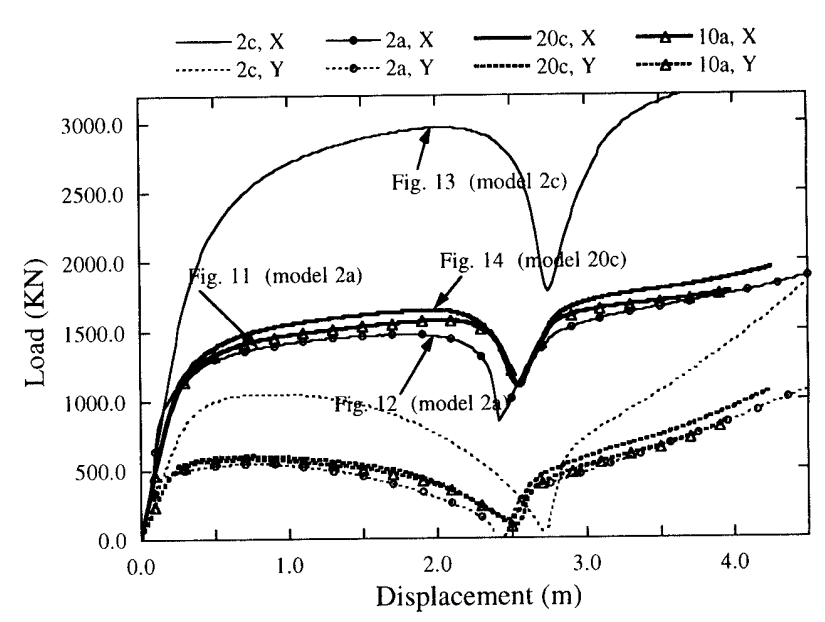

Fig. 15 Load versus displacement at the loading point of the $3 \mathrm{D}$ portal frame

\section{Collapse Analysis of a Three-Dimensional Framed Structure}

The proposed element combined with the ASI technique is applied to the collapse analysis of a threedimensional portal framed structure that models a building. Figure 10 illustrates the configuration of the structure. The lateral displacement indicated by an arrow in the figure is prescribed incrementally. Columns in the first layer are fixed to the ground. Material properties and sizes are shown in Table 2. Analysis methods and numbers of subdivision are illustrated in Table 3 . Number of degrees of freedom of models $2 \mathrm{c}$ and $2 \mathrm{a}$ is 468 , that of model $10 \mathrm{a}$ is 3060 and that of model $20 \mathrm{c}$ is 9900 .

Deformed configurations of the models in Table 3 are shown in Figs. 11 to 14 . Figure 15 illustrates the 
load-displacement curves at the loading point. The curves for $X$ direction and $Y$ direction of each model are illustrated. The loads first increase as the prescribed displacement increases, and then decrease. After that they increase again, since the third floor is completely collapsed. The result of model $2 \mathrm{c}$ is much stiff comparing with the other models. The results of models $2 \mathrm{a}, 20 \mathrm{c}$ and $10 \mathrm{a}$ almost agree with each other. In Figs. 12 and 14, the third floor is deformed largely. In Fig. 13, however, the concentrated deformation of the third floor is not observed because model $2 \mathrm{c}$ cannot express precise positions of the plastic hinges, thereby the rigidity of the columns of the third floor is overestimated.

\section{Concluding Remarks}

The ASI (Adaptively Shifted Integration) technique is applied to the degenerated element proposed by Dvorkin et al. that considers large displacements/ rotations. Numerical experiments show that when the ASI technique is applied to the original Dvorkin's element, a kind of locking occurs as the rotations increase. A method to avoid this locking is proposed; i.e., the directors of the element are redefined in each incremental step to be perpendicular to the neutral axis. Features of the proposed element are (1) arbitrary large rigid rotation about a plastic hinge that is located at an arbitrary position can precisely be considered, and ( 2 ) when finely subdivided model is used, the result obtained by the element with the ASI technique agrees with the result obtained by the element without the ASI technique. Some other elements that are almost equivalent to the proposed element are also shown, to which the ASI technique can be applied.

As an illustrative example, a three dimensional portal framed structure is analyzed. The results show that by using the proposed element with the ASI technique, precise collapse analysis considering large displacements/rotations can be performed if each member of the structure is subdivided into at least two elements.

\section{References}

(1) Toi, Y., Shifted Integration Technique in Onedimensional Plastic Collapse Analysis Using Linear and Cubic Finite Elements, Int. J. for Num. Meth. in Engin., Vol. 31(1991), p. 1537-1552.

(2) Toi, Y. and Isobe, D., Adaptively Shifted Integration Technique for Finite Element Collapse Analysis of Framed Structures, Int. J. for Num. Meth. in Engin., Vol. 36 (1993), p. 2323-2339.

(3) Toi, Y. and Yang, H., Simulation of Collapse of Framed Structures(Part 1), J. Soc. Naval Architects Japan(in Japanese), No. 166(1988), p. 285-
294.

(4) Toi, Y., Yang, H. and Obata, K., Simulation of Collapse of Framed Structures(Part 1), J. Soc. Naval Architects Japan(in Japanese), No. 167 (1989), p. 169-177.

(5) Toi, Y. and Isobe, D., Finite Element Analysis of Buckling Collapse Behaviors of Framed Structures by Using Adaptively Shifted Integration Technique, J. Soc. Naval Architects Japan(in Japanese), No. 174 (1993), p. 469-477.

(6) Toi, Y. and Isobe, D., Earthquake Resistance Analysis of a Large-Scale Framed Structure by Using Adaptively Shifted Integration Technique, Seisan-Kenkyu(in Japanese), Vol. 46, No. 10 (1994), p. 5-8.

( 7 ) Isobe, D. and Toi, Y., Finite Element Analysis of Dynamic Collapse Behaviors of Brittle Framed Structures by the Adaptively Shifted Integration Technique, J. Soc. Naval Architects Japan(in Japanese), No. 180 (1996), p. 471-478.

(8) Toi, Y. and Saito, Y., Finite Element Collapse Analysis of Brittle Framed Structures by the Adaptively Shifted Integration Technique, Trans. Jpn. Soc. Mech. Eng., (in Japanese), Vol.62, No. 604, A (1996), p. 153-159.

(9) Miyamura, T., Toi, Y. and Haze, T., ElasticPlastic Analyses of Steel Damper by Using Degenerated Timoshenko Beam Element Based on the ASI technique, Seisan-Kenkyu(in Japanese), Vol. 49, No. 2(1997), p. 5-8.

(10) Miyamura, T., Toi, Y. and Haze, T., Geometrically Nonlinear Analyses Using the Degenerated Timoshenko Beam Element Based on the Adaptively Shifted Integration Technique, Proc. Conf. Comp. Eng. Sci., (in Japanese), Vol. 2, No. 2 (1997), p. 439-442.

(11) Dvorkin, E., Onate, E. and Oliver, J., On a Nonlinear Formulation for Curved Timoshenko Beam Elements Considering Large Displacement/Rotation Increments, Int. J. for Num. Meth. in Engin., Vol. 26 (1988), p. 1597-1613.

(12) Bathe, K.J. and Bolourchi, S., Large Displacement Analysis of Three-Dimensional Beam Structures, Int. J. for Num. Meth. in Engin., Vol. 14 (1979), p. 961-986.

(13) Bathe, K.J., Finite Element Procedures in Engineering Analysis, (1982), Prentice-Hall.

(14) Belytschko, T. and Hsieh, B.J., Non-linear Transient Finite Element Analysis with Convected $\mathrm{Co}^{-}$ ordinates, Int. J. for Num. Meth. in Engin., Vol. 7 (1973), p. 255-271.

(15) Meng, L., Ohi, K. and Takanashi, K., A Simplified Model of Steel Structural Members with Strength Deterioration Used for Earthquake Response Analysis, J. Struct. Contr. Eng., AIJ, (in Japanese), No. 437 (1992), p. 115-124.

(16) Hisada, T. and Noguchi, H., Foundation and Application of Nonlinear Finite Element Method, (in Japanese), (1995), Maruzen.

(17) Noguchi, H. and Hisada, T., Integrated FEM 
Formulation for Total/Updated-Lagrangian Method in Geometrically Nonlinear Problems, JSME Int. J., Series I, Vol. 38, No. 1 (1995), p. 2329.

(18) Simo, J.C., et al., On a Stress Resultant Geometrically Exact Shell Model. Part III, Comp. Meth. in Appl. Mech. Engin., Vol. 79 (1990), p. 21-
70.

(19) Miyamura, T. and Toi, Y., Analyses of Three Dimensional Framed Structure by Using the Degenerated Beam Element Based on the ASI Technique, Sum. Tech. Papers, Annul. Meet. AIJ, B1(in Japanese) (1997), p. 377-378. 